\section{Atemwege stabil, Haut nicht}

\section{Seit Jahrzehnten nehmen weltweit die allergischen Erkrankungen bei Kindern zu. Aktuelle epidemiologische Daten aus der Schweiz zeigen Unterschiede bei den Organmanifestationen und bei den Geschlechtern.}

D as multizentrische „Swiss Surveillance Programme on Childhood Allergy and Respiratory Symptoms with Respect to Air Pollution" (SCARPOL) untersucht seit 1992 die Entwicklung von Atemwegserkrankungen und allergischen Erkrankungen bei Schweizer Kindern. Dazu werden in drei Städten und vier ländlichen Bereichen im Rahmen von Routinebesuchen in Kindergärten und in der 1., 4. und 8. Schulklasse regelmäßig Datenerhebungen durchgeführt. Grundlage der Dokumentation ist der ISAAC-Fragebogen.

In dieser Zeit von 1992 bis 2001 nahm die Zahl der von einem Heuschnupfen betroffenen Elternteile zu, ebenso der Anteil der gestillten Kinder und der Haushalte, die ein Haustier wegen einer Allergie abgeschafft hatten. Abgenommen haben während dieses
Zeitraums unter anderem die Schimmelbelastung, die Anzahl der mit Teppichen ausgestatteten Schlafzimmer und die Häufigkeit der Haustierhaltung

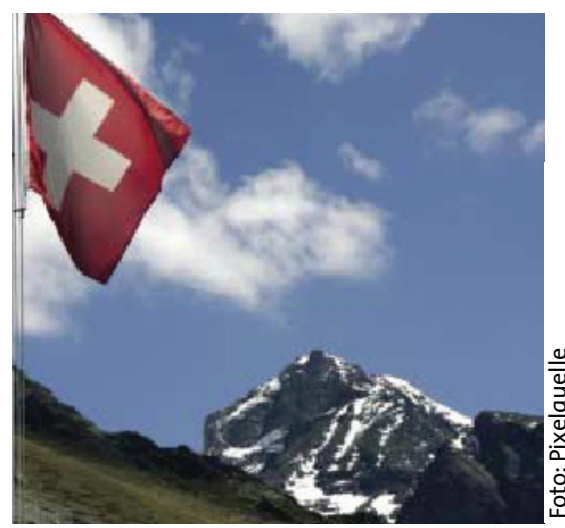

Gipfel erreicht? In der Schweiz nimmt die Prävalenz allergischer Atemwegserkrankungen nicht mehr weiter zu.

\title{
Stillverhalten bestimmt Stillerfolg
}

\section{Stillen wird als Präventionsmaßnahme gegen Allergien allgemein empfohlen. Doch in einigen Studien ergab sich auch ein erhöhtes Asthma- und Ekzemrisiko bei gestillten Kindern. Das könnte am Ver- halten der Mütter liegen.}

U $m$ herauszufinden, ob frühe Zeichen einer Atopie das Stillverhalten von Müttern verändern, führten Epidemiologen von der University of Melbourne eine prospektive Kohortenstudie mit 620 Kindern durch. In den ersten 64 Lebenswochen der Säuglinge wurden die Mütter alle vier Wochen telefonisch befragt, anschließend noch einmal in der 78. und 104. Woche, also mit eineinhalb und zwei Jahren. Die Frauen sollten jeweils Angaben zur Ernährung des Kindes sowie zu ersten Anzeichen von atopischen Erkrankungen wie Ekzemen oder Wheezing ma- chen. Außerdem wurden im Alter von sechs und zwölf Monaten Pricktests durchgeführt.

Nur $8,4 \%$ der Kinder wurden gar nicht gestillt, weitere $25 \%$ nicht ausschließlich. Ein Ekzem als häufigstes erstes Zeichen einer Atopie wurde bei etwas mehr als einem Drittel $(34,2 \%)$ der Kinder berichtet. Für Kinder, die mindestens zwei Wochen ausschließlich gestillt worden waren, zeigte sich, dass frühe Zeichen einer atopischen Erkrankung oder ein positives Pricktestergebnis mit einer um $28 \%$ verringerten Wahrscheinlichkeit für die Beendigung sowie der Exposition der Kinder gegenüber Tabakrauch durch die Mutter. Die Häufigkeit von allergischem Asthma und allergischer Rhinitis bei den Kindern nahm über den Beobachtungszeitraum hinweg leicht ab. Die Asthmaprävalenz reduzierte sich von $8,1 \%$ im Jahr 1992 auf 7,5\% im Jahr 2001, die Heuschnupfenprävalenz entsprechend von $6,4 \%$ auf $5,6 \%$. Dagegen nahmen die Berichte über ein atopisches Ekzem während dieses Zeitraums stetig zu: von 4,6\% im Jahr 1992 bis auf 7,6\% 2001. Betroffen von der signifikanten Zunahme der Neurodermitis waren vor allem die Mädchen.

Fazit: Daten aus der Schweiz deuten darauf hin, dass die Prävalenz von Asthma und allergischer Rhinitis in Mitteleuropa möglicherweise ein Plateau erreicht hat. Dagegen steigt die Häufigkeit der atopischen Dermatitis bei Schulkindern weiter an, vor allem bei den Mädchen.

Grize L et al. Trends in prevalence of asthma, allergic rhinitis and atopic dermatitis in 5-7-year old Swiss children from 1992 to 2001 . Allergy 2006; 61: 556-62

des ausschließlichen Stillens assoziiert waren. Das ausschließliche Stillen war außerdem dann länger, wenn die Mutter selbst eine Nahrungsmittelallergie hatte.

Die Ergebnisse können erklären, warum in Studien teilweise eine längere Stilldauer mit einer höheren Atopieprävalenz verbunden ist: Besorgte Mütter, insbesondere mit eigener allergischer Anamnese, stillen häufiger und bei ersten Zeichen einer Allergie auch länger als Mütter nicht betroffener Kinder.

Fazit: Frühe Zeichen einer allergischen Erkrankung im Säuglingsalter können $\mathrm{zu}$ einer verlängerten Stilldauer führen und damit die protektive Wirkung des Stillens in Studien maskieren.

$f k$

Lowe AJ et al. Atopic disease and breastfeeding - cause or consequence? J Allergy Clin Immunol 2006; 117: 682-7 